\title{
El papel de la mujer Emiratí en la política antes y después de la unión de los EAU
}

\author{
Noura Alkarbi - Universidad de Granada (D)0000-0002-2452-7283
}

Recepción: 01.04 .2020 | Aceptado: 30.06.2020

Correspondencia a través de ORCID: Noura Alkarbi

iD 0000-0002-2452-7283

Citar: Alkarbi, N (2020). El papel de la mujer Emiratí en la política antes y después de la unión de los EAU. REIDOCREA, 9, 138-154.

Financiación: Beca del Ministerio de Asuntos Presidenciales, Emiratos Árabes Unidos

Resumen: En el presente artículo pretendemos abordar la situación de la mujer emiratí antes y después de la unión de los Emiratos Árabes Unidos (EAU) en distintos ámbitos de la vida pública (social, económica, política y educativa), además de analizar qué avances han experimentado las mujeres en cada uno de esos ámbitos, con un especial hincapié en la representación política femenina actualmente en EAU. Los datos recopilados para este trabajo nos muestran una evolución respecto del reconocimiento del estatus de la mujer emiratí, lo que se podría interpretar como un avance importante hoy en día que ha permitido a los EAU en solo una generación situarse entre los primeros países árabes con mayor promoción del papel de la mujer, véase el informe de desarrollo humano del programa de las naciones unidas PNUD (2013). Los resultados obtenidos, si se mantiene la evolución favorable, apuntarían hacia un futuro prometedor en el que la mujer puede tener las mismas oportunidades que los hombres en todos los ámbitos de la vida política.

Palabras clave: Mujer Emiratí, Estado Actual, Política

\section{Emirati woman's role in politics before and after the unity of the United Arab Emirates}

Abstract: In this article, we intend to tackle the situation of the Emirati woman before and after the unity of the United Arab Emirates (UAE) in the different aspects of the public life: (social, economic, political and educational), in addition to the analysis of woman's achievements in all these fields, with special focus on the woman's political representation in UAE. The data collected for this work shows a development in the acknowledgment of the Emirati woman's condition which can be construed as an important development to date which allowed UAE, in a single generation, to be ranked as one of the first Arab countries to promote woman's role. See the United Nations Development Programme (UNDP) report of human development 2013. The obtained results - in a case a favorable development is maintained - aim towards a promising future in which woman can have the same opportunities as men in all fields of the political life.

Keyword: Emirati Woman, Current Situation, Politics

\section{Introducción}

No hay duda de que las mujeres desempeñan un papel esencial en el crecimiento y prosperidad de cualquier sociedad. En el caso de los EAU, el conjunto de costumbres y tradiciones predominantes influyen en la posición que desempeña la mujer emiratí. Las mujeres en los EAU se distinguen de otras mujeres de otras sociedades debido a factores económicos y sociales, sin olvidar la influencia de las creencias, costumbres y tradiciones, que son muy relevantes (Al Mazrouei, 2015:15) ${ }^{1}$.

Podemos dividir la población de los EAU en dos grupos fundamentales, si atendemos a dos corrientes históricas por el origen y la estructura demográfica:

- Beduino: este viene caracterizado por el movimiento de un lugar a otro y subsiste principalmente del pastoreo.

\footnotetext{
${ }^{1}$ Sultan Al Mazrouei, Maryam, Zayed and Women's Education en Abu Dhabi, Archivos Nacionales, Ministerio de Asuntos Presidenciales, Publicación y Distribución de Dar Kuttab, Primera Edición, Emiratos Árabes Unidos, 2015, p. 15.
} 
- Urbano: compuesto por una población muy diversa y con diferentes estilos de vida: agricultores, pescadores, buscadores de perlas, comerciantes y artesanos. Cada grupo tiene sus características socioeconómicas, que son las que determinan el papel y la posición de la mujer (Centro de Documentación e Investigación de EAU, 2004:19)².

Por lo tanto, conviene destacar la situación de la mujer en el período previo al auge del petróleo y al establecimiento de EAU, el 2 de diciembre de 1971. Señalamos algunos aspectos fundamentales en los ámbitos sociales, económicos y educativos, que son claves para el desarrollo de la sociedad y de los cuales realizaremos un análisis pormenorizado.

\section{En el ámbito social}

En el período previo al auge del petróleo y al establecimiento de EAU, el 2 de diciembre de 1971 El papel de la mujer se limitaba al cuidado familiar integral en ausencia del hombre, cabeza de la familia, y a tomar en su nombre las decisiones pertinentes. A pesar de las limitaciones económicas y educativas que prevalecían en la época, las mujeres emiratíes gozaban de un gran respeto en la sociedad tradicional de los EAU. Este respeto viene impuesto por la aplicación de los derechos y recomendaciones que les reconocía el Islam (Sayegh, 2017:25). ${ }^{3}$

Durante ese período, debido paradójicamente a las limitaciones económicas, las mujeres emiratíes desempeñaban funciones sociales y económicas más activas, en ausencia de los hombres, que hacían a la mar en busca de perlas y pasaban largos períodos de ausencia del hogar familiar. En esta época, caracterizada por un gran dinamismo y actividad de la mujer en la vida pública, las mujeres contribuían al incremento de los ingresos familiares y desempeñaban otros papeles importantes, Estos roles los desempeñó en ausencia del varón (padre, esposo o hijo), lo que le confirió a la mujer el doble papel de padre y madre en las siguientes áreas (NABAWI, 1997:464).

1. Educación de los menores a cargo, su preparación y capacitación para el futuro. Dependiendo del género del menor, se le orientaba hacia una opción de formación; el niño, por ser varón, se le instruía en destrezas y habilidades profesionales; y a la niña se les enseñaban las tareas del hogar y la educación de los hijos. Este reparto es el reflejo de los roles educativos que socialmente se asignaban a hombres y mujeres en esta época anterior a la fundación de EAU y al descubrimiento de los hidrocarburos.

2. La garantía de seguridad y protección a los miembros de la familia. Dicha responsabilidad requiere dureza, vigilancia, resolución y fuerte determinación para poder hacer frente a ciertas situaciones que pueden darse.

3. Control y supervisión de los diferentes acontecimientos familiares, ya sea en la celebración de matrimonios, en el empleo o en la resolución de conflictos entre miembros de la misma familia, o entre familias.

\footnotetext{
2 Centro de Documentación e Investigación, Zayed and Women, Primera Edición, Abu Dhabi, 2004, p. 19.

3 SAYEGH, F., Mujeres de los Emiratos Árabes Unidos bajo la Unión: Logros y aspiraciones. Centro Masabar de Estudios e Investigaciones, Dubai, 2017.p.25

${ }^{4}$ Al-NABAWI, M., Jubileo de Plata y Renacimiento de la Mujer, Unión General de Mujeres, Abu Dhabi, Emiratos Árabes Unidos, 1997, p. 46
} 


\section{En el ámbito económico}

La actividad económica durante este período se basaba principalmente en la pesca y en el comercio de perlas obtenidas mediante el método tradicional del buceo. En este sentido, se dan casos de mujeres que tienen en su propiedad barcos dedicados a estos oficios. No obstante, a pesar de que se dan casos de mujeres comerciantes, lo cierto es que en muchos casos su trabajo venía supervisado por hombres. De este modo, las mujeres que desarrollaban su actividad en estos sectores podían llegar a obtener un lucro importante, en especial las mujeres de la familia gobernante.

El descubrimiento del petróleo trajo consigo una gran transformación social, ya que se convirtió en el principal activo económico del país. El precio de los terrenos donde se desarrollaba la actividad petrolífera aumentó considerablemente y, con él, la riqueza de las mujeres propietarias.

Asimismo, la aparición de perlas artificiales provenientes de otros países golpeó directamente este sector en EAU, por lo que la región sufrió una crisis económica importante, tras lo cual, las mujeres se vieron obligadas a depender exclusivamente de la ganadería como primer recurso económico. Además, empezaron a realizar tareas propias de la sociedad beduina (tejer lana, apicultura, etc.). En cuanto a las mujeres en las comunidades agrícolas, estas se ocupaban del cultivo, regadío y cosecha (ALQASIMI, 1980:66) ${ }^{5}$. Por lo cual, las mujeres tenían más posibilidades que los hombres de obtener ingresos en tiempos de dificultades económicas (USRA SOFFAN, $1980: 66)^{6}$.

Las costumbres y tradiciones de la sociedad no impidieron a las mujeres contribuir económicamente, a través de la realización de actividades mercantiles, como la venta de sus productos y de su cosecha. Esto ocurre en los años cincuenta del siglo pasado, donde las mujeres tenían una fuerte presencia en el mercado emiratí de compraventa de productos y transacciones (AL-SHAMSI, 2015:38) ${ }^{7}$.

\section{En el ámbito político}

Antes de hablar del papel desempeñado por la mujer emiratí hoy día en la esfera política, conviene sacar a colación los orígenes de la participación de las mujeres en la vida política en los EAU, lo que fundamenta su participación en la actualidad. Documentación británica hacen referencia al papel desempeñado por las Sheikhasen ${ }^{8}$,en la toma de decisiones políticas. En estos documentos, se hacía mención del gran papel desempeñado por la fallecida Sheikha Salama, madre del jeque Zayed bin Sultan Al Nahyan. Se caracterizaba por su fuerte personalidad y su voluntad decidida. Esto quedó patente durante los años que fueron marcados por eventos importantes a los que asistió y en los que se hizo constar su carácter. (ALGHAFLI.2011:330) ${ }^{9}$ Sheikha Salama ha dejado un impacto en la personalidad del jeque fallecido Zayed bin Sultan, ya que se dice que la gran sabiduría del jeque Zayed

\footnotetext{
${ }^{5}$ AL-QASIMI, H., "Mujeres en los Emiratos Árabes Unidos: desafíos de la educación, la acción y la toma de decisiones", Serie de documentos científicos, Sociedad social, Sharjah, Emiratos Árabes Unidos,1993, p. 34.

${ }^{6}$ USRA SOFFAN, L., Las mujeres en la U.A.E, Croom Helm, Londres, 1980, p 66.

${ }^{7}$ AL-SHAMSI, Najib., "La mujer y el desarrollo en Ras Al Khaimah", Al Masar para Estudios Económicos y Publicaciones, Primera Edición Ras Al Khaimah, Emiratos Árabes Unidos, 2015 p. 38.

${ }^{8}$ Altas jerarcas mujeres.

${ }^{9}$ MOHAMMED SAIF AL GHAFLI, H., Participación política de las mujeres en los Emiratos Árabes Unidos (Nuevo Testamento para las mujeres emiratíes), Dar Al-Nahda Al Arabiya, Primera edición, 2011, pp. 330-331.
} 
"la heredó de su madre". También, hallamos documentos que recogen el papel desempeñado por la difunta Sheikha Hessa bint al-Murr, la esposa del difunto jeque Saeed Al-Maktoum, en la vida política del Emirato de Dubai entre los años 1930 y 1940 (ALGHAFLI .2011:331) ${ }^{10}$.

El escritor Belgrave menciona en su libro "La costa pirata" la batalla que tuvo lugar entre los hijos de Ras Al Khaimah, liderados por los Qawasm y los ingleses; donde la ciudad de Ras al-Khaimah fue asediada, bombardeada y quemada en 1819 (y esta no es la primera vez que se bombardea y se quema esta ciudad histórica). Balgreif añade sobre este hecho lo siguiente: "Poco antes de la medianoche, escuché disparos de baterías de artillería, seguidos de muchos disparos. En ese momento, los piratas parecían estar intentando lanzar un ataque sorpresa desde la punta de la ciudad, parecían estar arrastrándose sobre sus estómagos desde la orilla hasta que alcanzaron detrás de las armas, atacaron a los guardias y mataron a un grupo de soldados con el coronel jefe de este grupo avanzado. Los soldados avanzaron al sitio de batalla, obligando al enemigo a huir después de que muchos piratas murieron, y llevando sus heridos con ellos. Muchos de los heridos eran mujeres que participaron en el ataque. Las mujeres de los Qawasim eran tan valientes como los hombres" (ALSHAMSI, 2015:26-27) ${ }^{11}$.

Este es un ejemplo de la lucha y el activismo que en tal batalla tuvieron las mujeres emiratíes. Los hechos quedaron recogidos en la crónica que se narra esta obra.

\section{En el ámbito educativo}

En relación con la educación moderna ${ }^{12}$, los EAU se han visto afectados por diferentes factores históricos y políticos lo que hizo que la educación sea prácticamente inexistente tanto para hombres como para mujeres en épocas anteriores. El inicio del movimiento educativo en los EAU se vio en las décadas de 1950 y 1960, dónde influyeron algunos países árabes. (THABET: 1983:60) ${ }^{13}$ La relación de Gran Bretaña

${ }^{10}$ MOHAMMED SAIF AL GHAFLI, H., Participación política de las mujeres en los Emiratos Árabes Unidos
(Nuevo Testamento para las mujeres emiratíes), Dar Al-Nahda Al Arabiya, Primera edición, 2011, pp. 330-331.

${ }^{11}$ AL-SHAMSI, N.: "La mujer y el desarrollo en Ras Al Khaimah", Al Masar para Estudios Económicos y Publicaciones, Primera Edición Ras Al Khaimah, Emiratos Árabes Unidos, 2015, pp. 26-27.

${ }^{12}$ La educación moderna se define como la transmisión del conocimiento por parte de un enseñante a un individuo o grupo de individuos denominados aprendices, considerados como organismos inteligentes con capacidades para adquirir, vivenciar y experimentar los conocimientos, además de desarrollar sus capacidades y habilidades para actuar en su medio social y realizar transformaciones sobre su realidad.

La educación moderna se basa en el método científico, que nos enseña a pensar-actuar-predecir y resolver. La interacción entre los alumnos es muy importante, pues el trabajo en equipo permite el intercambio de ideas, la cooperación y el desarrollo de habilidades como la tolerancia y el respeto.

En la educación moderna las herramientas de aprendizaje son: los sentidos, las vivencias de la vida real y las experiencias del aprendiente. Los libros continúan siendo una base de apoyo e información, susceptible de ser cuestionada y analizada, o incluso rebatida y desechada por los aprendientes. Se han incorporado a la educación moderna actividades de socialización del conocimiento, trabajo en equipo, desarrollo de proyectos y dinámicas grupales, además de herramientas tecnológicas como el acceso a la información y su transmisión vía electrónica.

13 THABET, N.: Mujeres, desarrollo y cambios sociales asociados. Un estudio de campo en los EAU, Dar Al Sallas, Kuwait, 1983, p. 60. 
con los jeques era diferente al resto de las colonias, ya que no introdujo cambios económicos y sociales en la zona, y siguió con la enseñanza tradicional en las mezquitas, consideradas como el único lugar donde estudiaban los niños, memorizaban el Corán y aprendían la lectura y las matemáticas.(USRA SOFFAN.1980:68). ${ }^{14}$

Esto siguió así hasta el surgimiento de la educación formal como resultado del renacimiento cultural árabe. La fundación de las escuelas privadas fue el resultado de los esfuerzos de los comerciantes e intelectuales de los EAU, No es hasta 1953 cuando se introdujo por primera vez la educación formal en el Emirato de Sharjah, con la apertura de la primera escuela masculina, seguida de una escuela femenina en 1954 (KHALIFA, 1996:36) ${ }^{15}$ como resultado de la implicación de intelectuales de este emirato. La primera escuela femenina llevó el nombre de "Escuela de primaria AlZahraa para niñas", la primera de su género. En el curso académico 1955-1956, el número de alumnas fue 30 (AL-QASIMI, 1988:137 ${ }^{16}$. Más tarde, se abrieron más escuelas femeninas en los EAU (KHALIFA, 1996:50) ${ }^{17}$.

Del papel desempeñado por las mujeres en los EAU antes de la unión, podemos concluir que se trata de un conjunto de roles, como madre y ama de casa en el sistema familiar y como actora en el sistema económico con un nivel académicoformativo bajo. Sus valores derivan de la religión musulmana y de las tradiciones árabes (ABDEL, 2017:83) ${ }^{18}$ La participación de las mujeres emiratíes en este período fue relevante teniendo en cuanta las responsabilidades que desempeñaban. Sin embargo, la aparición del petróleo frenó el desarrollo de las actividades realizadas por ciertas mujeres anteriormente.

Esta observación fue citada por Yvon Haddad, investigadora estadounidense, en su análisis en el que dice: "Para las mujeres en el Golfo, noté que la década de 1950 se caracterizó por la participación general de las mujeres en el campo de trabajo, así como en la década de 1960. Sin embargo, en los países del Golfo en particular, en la década de 1970 y hasta ahora, esta participación se ha reducido, debido al hecho de que la prosperidad económica a medida que se expande disminuye el número de mujeres trabajadoras" (AL-QASIMI, 1993:108) ${ }^{19}$.

Con la cristalización de la Unidad Federal en sus instituciones sociales, económicas y culturales y en vista de las orientaciones políticas del estado federal para activar el papel de las mujeres en el proceso de desarrollo, por un lado, y la multiplicidad de campos de trabajo y producción por otro, el gobierno federal ha creado leyes que permiten la participación de las mujeres de forma igualitaria a los hombres.

El sistema legislativo dedica muchos artículos como la Ley Federal № 8 de 1980 relativa a la regulación de las relaciones laborales tuvo en cuenta la privacidad de la

\footnotetext{
${ }^{14}$ USRA SOFFAN, L., Las mujeres en la U.A.E, Croom Helm, Londres, 1980, p. 68.

${ }^{15}$ KHALIFA, A., El papel educativo y cultural de las sociedades de mujeres en los Emiratos Árabes Unidos, Un estudio de evaluación, El Comité de Definición, Composición, Traducción y Publicaciones, Emiratos Árabes Unidos,1996, p. 36.

${ }^{16}$ AL-QASIMI HIND, AA., "La constante y la variable en la cultura de las mujeres en los Emiratos Árabes Unidos", Sociedad de Socialistas, Primera edición, Sharjah, 1988, p. 137.

${ }^{17}$ KHALIFA, A., Papel educativo y cultural de las asociaciones de mujeres en los Emiratos Árabes Unidos, 1996, p. 50.

${ }^{18}$ ABDEL MUTTAL, N.: Mujeres emiratíes entre identidad y modernidad, Oficina del conocimiento árabe, primera edición, 2017, p. 83.

${ }^{19}$ AL-QASIMI HIND, "Mujeres en los EAU: Desafíos de la educación, la acción y la toma de decisiones", 1993, op. cit., p. 108.
} 
naturaleza innata de las mujeres al enfatizar la necesidad de preservar la seguridad de las mujeres a través de la declaración de trabajos en los cuales las mujeres no pueden ser empleadas de acuerdo con el Artículo 29; al prohibir el empleo de mujeres en trabajos forzados, peligrosos o perjudiciales para la salud o la moral. Las horas y los horarios de trabajo se regularon de acuerdo con el Artículo 27, siempre que las mujeres no trabajen por la noche. La trabajadora también recibe unas vacaciones de parto de acuerdo con el artículo 30 y las horas de lactancia están reguladas en el artículo 31. Según el artículo 1 y 2, los establecimientos están obligados a establecer guarderías para los hijos de mujeres empleadas de acuerdo con el decreto ministerial núm. 19 de 2006 sobre guarderías en ministerios e instituciones garantiza la protección y seguridad a las mujeres en su lugar de trabajo, además de precisar que el trabajo es un derecho de las mujeres tal como lo es para el hombre. El empleo de las mujeres también ha sido regulado tomando en consideración la multiplicidad de roles llevados a cabo por la mujer, tratando de conciliar su trabajo como empleada y madre (Centro de Documentación e Investigación.2014:29). ${ }^{20}$

En esta misma línea, el Ministerio de Educación como una entidad federativa, ha realizado grandes esfuerzos para difundir la educación entre las mujeres, con el acceso a la educación en todo el país y creando universidades. También el Estado se mostró dispuesto a apoyar a la mujer para la realización de sus estudios de posgrado y la obtención de un doctorado en las diferentes disciplinas ofreciéndoles la posibilidad de estudiar en otras universidades árabes y extranjeras (AL-GHAFLI, 2011:88) ${ }^{21}$.

\section{La realidad actual de las mujeres emiratíes y los horizontes futuros}

Conviene realizar una lectura sobre la realidad de las mujeres emiratíes con el fin de comprender y analizar el proceso propio de transformación, así como el cambio social, considerado como un factor que sirve esta realidad y la promueve hacia un futuro que se adapta al proceso de desarrollo que tiene lugar en los EAU en muchos aspectos de la vida.

De ahí que sea necesario entender la realidad de las mujeres de los EAU objetiva y científicamente para facilitar al investigador la tarea de analizarla con precisión. Por eso, esta parte de nuestro trabajo tiene por objetivo arrojar luz sobre la representación política de las mujeres en los EAU.

Para que nuestra presentación sea objetiva e inclusiva, nos parece apropiado emplear todas las cifras, estadísticas y tablas disponibles sobre la realidad de las mujeres emiratíes y su participación en las distintas áreas de la vida, tal y como se indica a continuación:

\section{1- Educación}

La educación se considera como un pilar fundamental de cualquier sociedad, y su desarrollo está vinculado a ella, además de ser una herramienta necesaria e importante en el desarrollo de otros ámbitos. Por esto la inversión en educación es una necesidad urgente para el cambio social en cualquier sociedad.

\footnotetext{
${ }^{20}$ Centro de Documentación e Investigación, (2004), Zayed and Women, Primera Edición, Abu Dhabi, p. 29.

${ }^{21}$ AL-GHAFLI, HAMDAN, M. S., Participación política de las mujeres en los Emiratos Árabes Unidos (Nuevo Testamento para mujeres emiratíes), Dar Al-Nahda Al Arabiya, Primera edición, 2011, p. 88.
} 
Al revisar las estadísticas, los registros y las tablas del cambio y del desarrollo de las mujeres emiratíes en el campo de la educación, encontramos que este movimiento se caracteriza por la permanencia y la continuidad. En este sentido, el desarrollo de la educación de las mujeres emiratíes avanza a un ritmo constante, continuo y estable.

Esto viene demostrado por las cifras proporcionadas por las instituciones responsables en estas cuestiones en EAU, o datos y estadísticas emitidos por organizaciones e instituciones de primera fila a nivel mundial. A través de estos datos, podemos observar cómo ha ido progresando el proceso de desarrollo de la mujer en los EAU en materia de la educación de las que recoge la legislación actual.

Por ejemplo, mediante el artículo 17 de la Constitución y el artículo 1 de la Ley Federal № 11 de 1972 sobre en carácter obligatorio de la educación en los EAU, podemos apreciar el impacto de la educación sobre el progreso de la sociedad. En esta misma línea, el país creó medidas para el impulso de la educación entre ellas hacer obligatorios los estudios de primaria y permitir a todos los aspirantes de estudias gratis en los niveles y en todo el país.

En julio de 2012, en lo que se refiere a la educación, el Consejo de Ministros creó una nueva ley compatible las existentes en muchos países desarrollados. Dicha ley estipula que la educación sea obligatoria para todos aquellos que hayan cumplido seis años, y que seguirá siendo obligatoria hasta que el alumno se gradúe del grado 120 cumpla los 18 años (Portal oficial del Gobierno de los EAU) ${ }^{22}$.

Esto contribuyó al incremento de la tasa de educación de las mujeres que superó las expectativas esperadas y, fruto de ello aumentó la participación de las mujeres en programas de educación pública, y su ocupación de cargos de prestigio en diversas universidades estatales, misiones, además de participar en cursos de capacitación.

Las estadísticas proporcionadas por las Naciones Unidas indican que los EAU es uno de los países más avanzados del mundo en materia de educación orientada hacia el perfil femenino. Las mujeres en los EAU han hecho un paso importante hacia la modernidad, dejando atrás muchas de las dificultades de la vida conservadora, para lograr una mayor prosperidad. A través del plan de educación llevado a cabo por el gobierno se superaron diferentes desafíos. La educación siempre ha sido una prioridad para el Estado, sobre todo al abordar los problemas concernientes las mujeres para convertir los EAU en uno de los mejores países árabes en este aspecto.

Los EAU han alcanzado un nivel avanzado entre otros países en el ámbito de la educación de las mujeres, ocupando el primer lugar con un 92\% correspondiente al porcentaje de mujeres graduadas que llegan a la universidad. Lo que hace que los EAU sean clasificados como uno de los países más avanzados a nivel nacional e internacional.

Los Emiratos han implementado diversas estrategias para establecer un sistema educativo avanzado, y el Estado ha garantizado una educación pública gratuita hasta la universidad para todos sus ciudadanos para difundir la educación en la sociedad y erradicar el analfabetismo.

También, el Gobierno está haciendo grandes esfuerzos para combatir el analfabetismo y lograr la igualdad de género en términos de tasas de alfabetización en los adultos.

\footnotetext{
22 Disponible en: https://www.government.ae/ar-AE/information-and-services/education Portal oficial del Gobierno de los EAU [Última consulta: 30/12/19].
} 
Gracias a estos esfuerzos, el indicador referente a mujeres adultas fue de aproximadamente $93 \%$ en 2005 comparado con $90 \%$ para hombres en el mismo año. La tasa de alfabetización de las mujeres fue ligeramente superior a la de los hombres que alcanzaron un $93.6 \%$ frente a las mujeres con un $97.0 \%$. Las tasas de analfabetismo han bajado de un $5 \%$ gracias a la puesta en marcha de centros de alfabetización para adultos.

El objetivo de los EAU fue el de eliminar las disparidades de género en la educación primaria y secundaria y en todos los niveles educativos, se ha conseguido en la enseñanza secundaria y universitaria. También, se ha trabajado para promover la educación superior, alentando el establecimiento de universidades públicas federales, locales y privadas. El número de mujeres en programas de educación superior fue del $71,6 \%$ del total de los estudiantes en universidades públicas y del $50,1 \%$ de los estudiantes en universidades e institutos privados.

La educación femenina a nivel universitaria ha suscitado el interés del Gobierno, puesto que el porcentaje de estudiantes mujeres en posgrado y maestría fue del $43 \%$ del total de estudiantes en el sector privado y del $62 \%$ en las universidades públicas, con un promedio del $46 \%$. Mientras que el porcentaje de graduados de bachillerato en instituciones de educación superior fue del $95 \%$ del número total de estudiantes en las universidades públicas y del $50,1 \%$ de los estudiantes en universidades e institutos privados.

Las mujeres también ocupan puestos docentes en los distintos niveles de educación a nivel nacional. Puesto que el porcentaje de maestras y maestros en el primer ciclo de educación básica en el país fue de aproximadamente $545.2 \%$. Esta tasa en el segundo ciclo de educación básica alcanza aproximadamente el $133.7 \%$ y se estima en $118.6 \%$ en la escuela secundaria, según el Centro Nacional de Estadísticas (AL-QUBAISI, 2014:6 $)^{23}$.

El presupuesto dedicado al sector educativo en el país, a saber, la educación pública, educación superior y becas universitarias para 2010 fue de 9.91 mil millones de dírhams (EAU). Comparado con los 6,34 mil millones de dírhams para el año 2005, donde el presupuesto reservado a la educación en el período 2005-2010 fue de 50.4 mil millones de dírhams.

En relación con la educación primaria y secundaria, el presupuesto dedicado fue de aproximadamente 17,2\% del presupuesto general del Estado en 2011, y 72,5\% del gasto total del sector educativo. El presupuesto para becas externas para 2010 ascendió a 272 millones a beneficio de 1755 estudiantes frente a 116 millones en 2005, y 9.91 mil millones en 2005, frente a 6.34 mil millones en 2005.

Todo ello contribuyó al aumento del número de alumnas en las escuelas estatales, un aumento del $28 \%$ en 2012 frente al año 2008. Además, las mujeres en los EAU consiguieron las mejores tasas de éxito académico, según el Informe elaborado por el Foro Económico Mundial 2012 (AL-QUBAISI, 2014:7)24 Y de este modo, el número de alumnas en las escuelas superó al número de hombres, a saber, que el $65 \%$ de los graduados universitarios en los EAU son mujeres, por lo que pasan a ser una referencia importante de talento para alcanzar los objetivos de crecimiento y desarrollo económico en los EAU.

\footnotetext{
${ }^{23}$ AL-QUBAISI, A., "Tendencias y políticas globales para la igualdad de género", Documento presentado a la Cumbre de Gobierno, DUBAI de octubre de 2014, pág. 6.

24 Ibid., p. 7.
} 
De lo dicho anteriormente, podemos observar que la mujer emiratí goza de plenos derechos que le permiten estudiar y trabajar en el ámbito de la educación. Todo esto se hace gracias a la Constitución que lo garantiza, así como a las estrategias y los planes establecidos por el Estado que se esfuerza para alcanzar dichos objetivos, entre ellos la igualdad de género. Los EAU han alcanzado las tasas más altas en este ámbito según estudios realizados por instituciones internacionales. A su vez, el Foro Económico Mundial para cerrar la brecha de género en 2016, indica que los EAU ocuparon el primer lugar en el subestándar de alfabetización (reports.weforum.org.2016) 25 .

\section{2- Salud}

Conviene precisar que la Constitución en los EAU garantiza a las mujeres una atención médica integral e incondicional. Según el artículo 19 de la Constitución: "la sociedad garantizará a los ciudadanos la atención de salud y los medios de prevención y tratamiento de enfermedades y epidemias, y alienta el establecimiento de hospitales, dispensarios y centros de tratamiento públicos y privados" (Constitución de los Emiratos Árabes Unidos $)^{26}$. En base a esto, los EAU han trabajado para establecer y desarrollar un plan sanitario gratuito en colaboración con el Ministerio de Salud.

También, los EAU se han centrado en lograr los objetivos de la Estrategia Nacional para la Maternidad y la Infancia. En cooperación con el Fondo de las Naciones Unidas para la Infancia (UNICEF) en la década que va del 2012 al 2021.

Todas las partes interesadas seguían muy de cerca las campañas de prevención o tratamiento de enfermedades. Entre todas ellas se destaca la Campaña de cáncer de mama, que fue patrocinada por Su Alteza Sheikha Fatima Bint Mubarak.

En 2002, Su Alteza Sheikha Fatima lanzó la Estrategia Nacional para la promoción de la Mujer en los EAU, cuyo objetivo fue el de mejorar la igualdad y el estatus de las mujeres. El informe del Foro Económico Mundial sobre la supresión de las disparidades entre los géneros de 2014 confirmó que en términos de atención médica se había conseguido la igualdad de género. El informe mostró una brecha inferior al $4 \%$ en la atención médica, lo que hace de los EAU uno de los países más activos en este sentido.

Los desarrollos económicos y sociales en los EAU han permitido mejorar las políticas sanitarias. Los programas de tratamiento y prevención implementados por el Ministerio de Salud han beneficiado mucho a las mujeres emiratíes, y como resultado de ello podemos ver que ha bajado la tasa de enfermedades, la tasa de mortalidad, especialmente entre los bebés, y ha aumentado la tasa de natalidad. Todo ello ha contribuido a una toma de conciencia por los ciudadanos.

Las estadísticas del Ministerio de Salud indican que la tasa de supervivencia femenina es de 78.6 años frente a 75.6 años a la masculina. La tasa bruta de natalidad fue de 1.62 por mil y la tasa de mortalidad infantil fue de alrededor de 7,5 por mil en 2007. Todas las políticas mencionadas anteriormente han contribuido a reducir la brecha de género.

\footnotetext{
${ }^{25} \mathrm{http}$ //reports.weforum.org/global-gender-gap-report-2016/economies/\#economy=ARE.

${ }^{26}$ Constitución de los Emiratos Árabes Unidos, Secretaría General del Consejo Nacional Federal, Dubai, 2015, pág. 11.
} 
Los EAU ocuparon el primer lugar en el subcriterio de GÉNERO (hombre / mujer) según el Foro Económico Mundial para cerrar la brecha de género para 2016 (reports.weforum.org.2016) ${ }^{27}$.

Las instituciones sanitarias en el país como el Ministerio de Salud, la Autoridad General de Servicios de Salud del Emirato de Abu Dhabi, el Departamento de Salud, los Servicios Médicos de Dubai, los servicios médicos en las fuerzas armadas y los servicios médicos en las compañías petroleras, además de las instituciones de salud en el sector privado, han desempeñado un papel importante ampliado su base de servicios para que llegue a todo el país. El concepto de atención sanitaria como un punto de acceso básico a los servicios de salud de calidad, tales como la atención terapéutica integral y la atención preventiva, reforzadora y rehabilitadora.

Por consiguiente, los perfiles estratégicos de salud en el país se basan en la prestación de atención médica integral en sus niveles iníciales de especialización para toda la población del país, especialmente orientados a la maternidad y a la infancia. Lo que se reflejó en la baja tasa de mortalidad materna debida a embarazos, partos por cada 100.000 mujeres en edad reproductiva 15-49 años, llegando la tasa a 2 - 4 hijos por mujer. Se registró una media de tres muertes por embarazos y partos en 2010 (Organización Mundial de la Salud.2015) ${ }^{28}$.

La erradicación de enfermedades transmisibles, especialmente las enfermedades infantiles objeto de estudio del programa nacional de inmunización y enfermedades de recién aparición y exóticas. La tasa de mortalidad de menores de cinco años por cada 1.000 nacidos vivos se redujo de 8,2 en 2011 a 7,6 en 2012, la tasa de mortalidad infantil por cada 1000 recién nacido disminuyó de 6,4 niños en 2011 a 5,9 niños en 2012 (Indicadores de salud para los Estados del CCG.2014:24) ${ }^{29}$.

La preparación de estrategias de salud y el establecimiento de controles y equipos sanitarios de alta calidad, acorde a las últimas tecnologías más innovadoras, han contribuido significativamente al desarrollo de condiciones sanitarias para las mujeres emiratíes y para la sociedad en general.

El Ministerio de Salud ha creado servicios de atención maternos e infantiles, mediante los cuales se puede seguir el estado de las mujeres embarazadas a través de centros y unidades maternas e infantiles en centros de salud de atención primaria, instituciones sanitarias y hospitales públicos especializados en obstetricia y ginecología.

De conformidad con la Constitución, las instituciones sanitarias en el país han desarrollado una gama de servicios con el fin de preservar la salud de las mujeres embarazadas, incluyendo el examen médico integral que abarca medición de peso, longitud y examen odontológico. El cuidado de la madre no se limita al período de embarazo, sino que se extiende hasta el período posparto. Pues, la madre visita el centro de salud para asegurarse de que no hay complicaciones relacionadas con el parto y también para apoyar y alentar la lactancia materna, así como examinar el estado de salud del recién nacido.

\footnotetext{
27 Ibid.

28 Organización Mundial de la Salud et al., "Tendencias en las tasas de mortalidad materna entre 1990 y 2015", publicadas en el sitio web de la FAO (www.who.int) 2015.

${ }^{29}$ El centro estadístico de los Estados del CCG, Indicadores de salud de los Estados del CCG, 2014. pág. 24.
} 


\section{3- Ámbito laboral, Parlamento y altos cargos}

Gracias a las políticas y estrategias adoptadas por los EAU para el desarrollo y el empoderamiento de las mujeres, estas han desempeñado un papel importante junto a los hombres para construir y contribuir al desarrollo de su país.

Todo esto ha llevado a las mujeres a ocupar puestos de trabajo en todas las áreas de la economía. Así como se ha demostrado que la presencia de la mujer en el mercado laboral tendría un impacto positivo y daría lugar a importantes ganancias económicas (ELBURG, 2017:14) ${ }^{30}$.

Desde el gobierno, se han centrado en invertir de forma real en la capacitación y formación de personas, en general y de las mujeres en particular. Además, han constatado que ante una mayor participación femenina se incrementaría el producto interno bruto en muchos países; en el caso de los EAU se ha incrementado en un $12 \%$ (ELBURG, 2017:5) $)^{31}$.

Con el fin de reducir la brecha de género y aumentar el empoderamiento de las mujeres, el Consejo de Ministros emitió un decreto, el 9 de diciembre de 2012, mediante el cual se exigía la representación del componente femenino en las juntas directivas de todos los organismos gubernamentales y empresas del Estado.

Se ha demostrado reiteradamente que las mujeres en los EAU son eficientes a la hora de ocupar diferentes cargos, y es por esta razón que se les ha permitido participar en las tomas de decisiones en todas las instituciones y organismos gubernamentales.

Los EAU son el segundo país en el mundo en implementar esta decisión, mejorando así el empoderamiento de las mujeres en el ámbito económico. Por otro lado, la Ley Federal № 9/2011 había aprobado el tipo de jornada laboral a tiempo parcial con un salario correspondiente al grado de trabajo, dando mayores oportunidades a las mujeres para que puedan conciliar su vida familiar con sus obligaciones profesionales.

En 2010, el Consejo de Ministros aprobó una decisión que otorgaba a las mujeres empleadas, con un esposo en paro, bonificaciones por hijos y vivienda. Teniendo en cuenta las circunstancias y condiciones de esta última se le otorgará un porcentaje de $60 \%$ por vivienda, y en caso de fallecimiento de su marido beneficiará de una baja de 4 meses y 10 días.

El Gobierno puso en marcha un sistema flexible para el trabajo de las mujeres, especialmente en los ministerios de carácter especializado en el que aplica el sistema de turnos, tratando además a los hijos de las ciudadanas como a los de los ciudadanos en los derechos y deberes (MANSOUR, 2015:12) ${ }^{32}$.

Todo ello ha contribuido a que las mujeres en los EAU llegasen a ocupar casi el 66\% de los puestos de trabajo en el sector gubernamental. El $30 \%$ de los cargos de liderazgo influyentes están en manos de mujeres. Las mujeres en los EAU han llegado a participar en la actividad económica local. El porcentaje de participación de las mujeres en el mercado laboral aumentó de $6.9 \%$ en 1986 a $13 \%$ en 1995 y $40 \%$ en

\footnotetext{
${ }^{30}$ ELBURG, C., et al.," Informe anual", Fondo Monetario Internacional, Washington, 2017, pág. 14.

31 Ibid., pág. 5.

32 MANSOUR, M. I., Derechos de las mujeres en los Emiratos Árabes Unidos, Unión General de Mujeres, Dubai, 2015, pág. 12.
} 
2009. Y con ello aumentó el número total de trabajadores en el país, del 8,9\% en 2006 al $9,1 \%$ en 2008.

De acuerdo con el informe emitido por el Foro Económico Mundial sobre la brecha de género para 2016, los EAU ocuparon el octavo lugar sobre igualdad de salarios por sector de trabajo. Todo esto no hubiera sido posible sin la decisión del Consejo de Ministros de formar el Consejo de Emiratos para el Equilibrio de Género en todos los campos de trabajo y contribuir a apoyar la posición de los EAU a nivel local e internacional, para lograr las expectativas marcadas por los EAU de estar entre los 25 primeros países en el índice de equilibrio de género en 2020 (reports.weforum.org.2016) ${ }^{33}$.

En cuanto a los altos cargos y las posiciones de liderazgo, las mujeres emiratíes han conseguido ocupar muchos de ellos y han alcanzado posiciones de liderazgo importantes en la sociedad gracias a los esfuerzos realizados en el marco del empoderamiento de las mujeres en las últimas décadas. Las mujeres han podido ocupar puestos judiciales, que siguen siendo reservados exclusivamente a hombres en muchos países del mundo. En 2007, se nombraron a dos fiscales para trabajar en el Departamento Judicial de Abu Dhabi. En 2008, una mujer fue nombrada jueza de tercera categoría en el Departamento Judicial de Abu Dhabi.

Por otro lado, las mujeres emiratíes han ocupado más del $60 \%$ de los empleos en el gobierno y el $30 \%$ de los altos cargos directivos en el sector gubernamental. El número de mujeres en todo el mundo es aproximadamente un de $48 \%$ (Revista Future Horizons, 2015:44) $)^{34}$, y el número de mujeres en el grupo de edad (15-24) años, es aproximadamente de $31,6 \%$ del número total de trabajadores en los EAU en 2011 (Revista Future Horizons, 2015:47) ${ }^{35}$

Las mujeres en los EAU han destacado en muchos ámbitos de trabajo no tradicionales, y han asumido cargos militares y judiciales. Su capacidad para liderar les dio la oportunidad de presidir las organizaciones regionales, como es el caso con la Organización de Mujeres Árabes (AL-QUBAISI, 2014:11) ${ }^{36}$.

En las instituciones sanitarias, las mujeres emiratíes ocupaban puestos de liderazgo. Las estadísticas muestran que el porcentaje de las mujeres que ocuparon altos cargos directivos en el Ministerio de Salud en el año 2013 fue de 47\%. Entre ellas está el 77\% de médicos, el $88 \%$ de dentistas, el $97 \%$ de farmacéuticos, el $94 \%$ de enfermeros y alrededor del 63\% del personal técnico (AL-SUWAIDI, 2013:1-11) ${ }^{37}$.

En el campo de los negocios y de la economía, la tasa de participación de las mujeres en los EAU alcanzó aproximadamente el 4,5\%. Además, participan en la administración del Departamento de Comercio e Industria en un 15\%, mientras alcanzó la participación de las mujeres en las pequeñas y medianas empresas aproximadamente un 30\%. Así como, consiguieron las mujeres empresarias un importe de ahorros que asciende a 36.7 mil millones de dírhams (alrededor de US \$10

\footnotetext{
33 http://reports.weforum.org/global-gender-gap-report-2016/economies/\#economy=ARE

${ }^{34}$ Revista Future Horizons, № 27 de julio y agosto de 2015, Dubai, pág. 44.

35 Ibíd., pág. 47

${ }^{36}$ AL-QUBAISI, A., Actitudes y políticas globales para la igualdad de género, Documento presentado a la Cumbre de Gobierno, Dubai, octubre de 2014, pág. 11.

${ }^{37}$ AL-SUWAIDI, J. S., "Umm Al-emirates Un modelo único de liderazgo mundial de mujeres", anexo especial de la revista Akhbar Al Saha, Emiratos Árabes Unidos, Dubai, septiembre de 2013, págs. 1-11.
} 
mil millones), sabiendo que Abu Dhabi cuenta con alrededor del $40 \%$ de todas las empresarias en el país (Unión General de Mujeres, 2014:4) ${ }^{38}$.

En el área del derecho de las mujeres a votar y ser electas para su representación en el Consejo Nacional Federal, esto se cumple de conformidad con las disposiciones del Artículo 70 (Constitución de los Emiratos Árabes Unidos ) ${ }^{39}$.

1. Ser ciudadano de uno de los Emiratos y residir permanentemente en el Emirato que representa en el Consejo.

2. No tener menos de veinticinco años.

3. Poseer la capacidad civil y una buena conducta, y no ser condenado por un delito que atente al honor a menos que sea rehabilitado de acuerdo con la ley.

4. Debe tener un buen dominio lingüístico tanto escrito como oral.

Esto les permitió a las mujeres presentarse y ser electas en todos los consejos legislativos. Así como desempeñó la Unión General de Mujeres, que fue fundada en 1975 e incluye a todas las asociaciones de mujeres en los EAU, un papel importante para promover la participación de las mujeres emiratíes en el proceso de desarrollo de los tres poderes legislativo, ejecutivo y judicial.

En 2008, la representación de mujeres en el Consejo de Ministros aumentó de dos a cuatro escaños, con cuatro ministras de los 22 miembros (EL-DIN, 2016:91) ${ }^{40}$. Además del nombramiento de dos mujeres como embajadoras en los EAU en el extranjero. En 2009, Sheikha Najla Al Qassimi y la Dra. Hessa Al Otaiba fueron designadas embajadoras ante el Reino de Suecia y España respectivamente, por primera vez en la historia del país. El número de mujeres embajadoras en los EAU aumentó en 2010 a tres embajadoras y un cónsul general.

Con respecto al papel de las mujeres en las elecciones, la Comisión Nacional Electoral de los EAU se estableció en 2006 para gestionar el primer proceso electoral en el país. Las mujeres desempeñaron un papel importante en la gestión electoral.

Dos de los siete centros de votación estuvieron bajo la dirección de dos mujeres. También había dos mujeres vicepresidentes en Sharjah y Ajman. Los jefes de los centros de votación de todo el país tenían un papel muy importante en el proceso electoral: eran responsables de supervisar el equipo de gestión electoral y el sistema de votación en cada centro para garantizar la transparencia del proceso, (ALDABBAGH, 2009:29) ${ }^{41}$.

El papel de la mujer en el Consejo Nacional Federal también se destacó durante el decimocuarto período legislativo en 2006, con la participación de nueve mujeres de entre un total de 40 miembros, lo que equivale al $23 \%$ de los escaños del Consejo. Este es el porcentaje más alto que se alcanzó en instituciones legislativas en cuanto a la representación de mujeres.

\footnotetext{
38 Unión General de Mujeres, Mujeres que Trabajan entre los Roles Sociales y los Requisitos Funcionales, Unión General de Mujeres, Dubai, abril de 2014, página 4.

${ }^{39}$ Constitución de los Emiratos Árabes Unidos, Secretaría General del Consejo Nacional Federal, Dubai, 2015, pág. 27.

4040 EL-DIN AHMED, W. H., "Empoderamiento político de las mujeres árabes", Centro de investigación prometedora en investigación social y estudios de la mujer, Riad, 2016., pág. 91.

${ }^{41}$ AL-DABBAGH. M. et al., "La experiencia parlamentaria de las mujeres emiratíes", Facultad de Dubai para Gestión Gubernamental, Dubai, 2009, pág. 29.
} 
Las mujeres miembros participaron en las actividades de supervisión interna del Consejo, así como lo hicieron de forma externa, a nivel de los parlamentos regionales e internacionales. También fueron nominadas en estas elecciones, como podemos apreciar en la siguiente tabla (AMIRI, 111:7) ${ }^{42}$ :

Tabla 1. Número de candidatos para las elecciones del Consejo Nacional Federal 2006

\begin{tabular}{|c|c|c|c|c|c|c|}
\hline Emirato & $\begin{array}{c}\text { Cuerpo } \\
\text { electoral } \\
\text { total }\end{array}$ & $\begin{array}{c}\text { Núm. de } \\
\text { mujeres } \\
\text { candidatas } \\
\text { inscritas }\end{array}$ & $\begin{array}{c}\text { Núm. de } \\
\text { hombres } \\
\text { inscritos } \\
\text { candidatos }\end{array}$ & $\begin{array}{c}\text { Núm. total de } \\
\text { candidatos } \\
\text { registrados }\end{array}$ & $\begin{array}{c}\text { Los } \\
\text { escaños }\end{array}$ & $\begin{array}{c}\text { Escaños } \\
\text { totales }\end{array}$ \\
\hline Abu Dhabi & 1741 & 14 & 86 & 100 & 4 & 8 \\
\hline Dubai & 1520 & 15 & 67 & 82 & 4 & 8 \\
\hline Sharjah & 1017 & 29 & 72 & 101 & 3 & 6 \\
\hline Ajman & 436 & 2 & 22 & 24 & 2 & 4 \\
\hline Umm Al Quwain & 403 & 1 & 28 & 29 & 2 & 6 \\
\hline Ras Al Khaimah & 1061 & 3 & 80 & 83 & 3 & 4 \\
\hline Fujairah & 417 & 1 & 36 & 37 & 2 & 40 \\
\hline Total & 6595 & 65 & 391 & 456 & 20 & 4 \\
\hline
\end{tabular}

Mediante esta tabla podemos observar el número de mujeres candidatas a las elecciones en 2006, desafortunadamente las cifras no indican un resultado muy satisfactorio como esperaban los líderes y los príncipes del Estado, hay que recordar que debido a que las elecciones se convocaron por primera vez en el Estado, la participación de las mujeres fue limitada, pero aceptable y esperamos que puede aumentar en las siguientes elecciones.

De hecho, la situación de las mujeres ha cambiado y su participación en las elecciones posteriores ha aumentado. En 2006, la participación de las mujeres en las elecciones fue de $14.25 \%$ del número total de candidatas. El porcentaje de las participantes en las elecciones en 2011 aumentó a 46\% con un total de 130 mil votantes en el segundo año de las elecciones en la Asamblea Nacional. Así como participaron unas 85 mujeres, con un porcentaje de $18 \%$, de un total de 468 candidatos en todos los emiratos del Estado. El número de mujeres en la Consejo Nacional Federal aumentó a siete mujeres con un $17.5 \%$ del número total de miembros. Conviene precisar que el nombramiento de la Dra. Amal Qubaisi como vicepresidenta del Parlamento, siendo la primera mujer en ocupar este cargo a nivel del Estado y del Consejo de Cooperación del Golfo, le confiere a esta sesión un carácter especial. ${ }^{43}$

El cambio realizado en los EAU estuvo marcado por un incremento del número de mujeres que ocupan cargos ministeriales, de cuatro pasando a ser ocho ministras, además de la investidura de la presidencia del Consejo Nacional Federal por una

${ }^{42}$ AMIRI, A. et al., "El papel de la mujer en el Consejo Nacional Federal", Consejo Nacional Federal, Dubai pág. 7.

${ }^{43}$ Fundación de Mujeres de Dubai, Gobierno de los Emiratos Árabes Unidos, Informe estadístico de mujeres, Emiratos Árabes Unidos, Dubai, 2013. pág. 3. 
mujer, como un paso pionero. Lo que indica un compromiso notable y un interés por parte de los EAU con respecto al tema del empoderamiento político de las mujeres. Como prueba de ello, podemos constatar, a partir del Informe de Desarrollo Humano del Programa de las Naciones Unidas para el Desarrollo (PNUD) del 2013, que los EAU han conseguido muchos logros en un tiempo record en materia de empoderamiento de las mujeres y reducción de las disparidades entre los géneros, lo que lo ubica en el puesto 41 de una lista de 187 países en todo el mundo. ${ }^{44}$

Como consecuencia de todo lo dicho anteriormente, podemos deducir que el futuro de las mujeres emiratíes depende de una serie de factores que pueden orientar este último hacia un cambio mejor. También por medio de este trabajo, muchas investigaciones y estudios locales anteriores, regionales e internacionales apuntan que todos los factores de la prosperidad de la mujer emiratí están en aumento, de manera que impulsa a las mujeres hacia el avance, el empoderamiento y el liderazgo, a pesar de la persistencia de algunos desafíos, particularmente en aspectos sociales y culturales en cuanto a los valores, costumbres y tradiciones sociales. ${ }^{45}$

En este sentido, el proceso de modernización requiere más esfuerzos y tiempo. Pero con los esfuerzos conjuntos de todos los líderes del Estado y bajo los auspicios especiales de los EAU, además de la combinación de los esfuerzos de las instituciones del estado y las instituciones de la sociedad civil y los medios de comunicación serán clave en la aceleración del proceso de cambio social y cultural de una manera que permita y impulse a las mujeres hacia el liderazgo y el avance.

Finalmente, conviene señalar que, partiendo de una metodología científicamente fundamentada para analizar y estudiar estos desafíos, estos pueden ser superados y, como dijo Su Alteza Sheikha Fatima Bint Mubarak, presidenta de la Unión General de Mujeres y presidente del Consejo Supremo para la Maternidad y la Infancia en los EAU.

44 MUTTAL, N. A., Empoderamiento de las Mujeres del Golfo, Oficina de Conocimientos Árabes, El Cairo, 2018., págs. 58-59.

${ }^{45}$ AL-GHARAIBEH, F. "Una exploración de la evolución de los roles de las mujeres en las sociedades del Consejo de Cooperación del Golfo" Fuente: Cuestiones de desarrollo social, Volumen 37, № 3, Consorcio Internacional para Desarrollo Social, 2015, pp. 22-44. 


\section{Referencias}

Sultan Al Mazrouei, Maryam, Zayed and Women's Education en Abu Dhabi, Archivos Nacionales, Ministerio de Asuntos Presidenciales, Publicación y Distribución de Dar Kuttab, Primera Edición, Emiratos Árabes Unidos, 2015.

Centro de Documentación e Investigación, Zayed and Women, Primera Edición, Abu Dhabi, 2004.

SAYEGH, F., Mujeres de los Emiratos Árabes Unidos bajo la Unión: Logros y aspiraciones. Centro Masabar de Estudios e Investigaciones, Dubai, 2017.

Al-NABAWI, M., Jubileo de Plata y Renacimiento de la Mujer, Unión General de Mujeres, Abu Dhabi, Emiratos Árabes Unidos, 1997.

AL-QASIMI, H., "Mujeres en los Emiratos Árabes Unidos: desafíos de la educación, la acción y la toma de decisiones", Serie de documentos cientíicos, Sociedad social, Sharjah, Emiratos Árabes Unidos, 1993

USRA SOFFAN, L., Las mujeres en la U.A.E, Croom Helm, Londres, 1980.

AL-SHAMSI, Najib., "La mujer y el desarrollo en Ras AI Khaimah", Al Masar para Estudios Económicos y Publicaciones, Primera Edición Ras Al Khaimah, Emiratos Árabes Unidos, 2015.

MOHAMMED SAIF AL GHAFLI, H., Participación política de las mujeres en los Emiratos Árabes Unidos (Nuevo Testamento para las mujeres emiratíes), Dar Al-Nahda Al Arabiya, Primera edición, 2011

MOHAMMED SAIF AL GHAFLI, $H$., Participación política de las mujeres en los Emiratos Árabes Unidos (Nuevo Testamento para las mujeres emiratíes), Dar Al-Nahda Al Arabiya, Primera edición, 2011.

AL-SHAMSI, N., "La mujer y el desarrollo en Ras Al Khaimah", Al Masar para Estudios Económicos y Publicaciones, Primera Edición Ras Al Khaimah, Emiratos Árabes Unidos, 2015.

THABET, N., Mujeres, desarrollo y cambios sociales asociados. Un estudio de campo en los EAU, Dar AI Sallas, Kuwait, 1983.

USRA SOFFAN, L., Las mujeres en la U.A.E, Croom Helm, Londres, 1980.

KHALIFA, A., El papel educativo y cultural de las sociedades de mujeres en los Emiratos Árabes Unidos, Un estudio de evaluación, El Comité de Definición, Composición, Traducción y Publicaciones, Emiratos Árabes Unidos,1996.

AL-QASIMI HIND, A. A.: "La constante y la variable en la cultura de las mujeres en los Emiratos Árabes Unidos", Sociedad de Socialistas, Primera edición, Sharjah, 1988.

KHALIFA, A., Papel educativo y cultural de las asociaciones de mujeres en los Emiratos Árabes Unidos, 1996.

ABDEL MUTTAL, N.: Mujeres emiratíes entre identidad y modernidad, Oficina del conocimiento árabe, primera edición, 2017.

AL-QASIMI HIND, "Mujeres en los EAU: Desafíos de la educación, la acción y la toma de decisiones", 1993.
Centro de Documentación e Investigación, Zayed and Women, Primera Edición, Abu Dhabi, 2004

AL-GHAFLI, HAMDAN, M. S., Participación política de las mujeres en los Emiratos Árabes Unidos (Nuevo Testamento para mujeres emiratíes), Dar Al-Nahda Al Arabiya, Primera edición, 2011.

AL-QUBAISI, A., "Tendencias y políticas globales para la igualdad de género", Documento presentado a la Cumbre de Gobierno, DUBAI de octubre de 2014.

Constitución de los Emiratos Árabes Unidos, Secretaría General del Consejo Nacional Federal, Dubai, 2015.

Organización Mundial de la Salud et al., "Tendencias en las tasas de mortalidad materna entre 1990 y 2015", publicadas en el sitio web de la FAO (www.who.int) 2015

El centro estadístico de los Estados del CCG, Indicadores de salud de los Estados del CCG, 2014.

ELBURG, C., et al.," Informe anual", Fondo Monetario Internacional, Washington, 2017.

MANSOUR, M. I., Derechos de las mujeres en los Emiratos Árabes Unidos, Unión General de Mujeres, Dubai, 2015.

Revista Future Horizons, № 27 de julio y agosto de 2015, Dubai.

AL-QUBAISI, A., Actitudes y políticas globales para la igualdad de género, Documento presentado a la Cumbre de Gobierno, Dubai, octubre de 2014

AL-SUWAIDI, J. S., "Umm Al-emirates Un modelo único de liderazgo mundial de mujeres", anexo especial de la revista Akhbar Al Saha, Emiratos Árabes Unidos, Dubai, septiembre de 2013

Unión General de Mujeres, Mujeres que Trabajan entre los Roles Sociales y los Requisitos Funcionales, Unión General de Mujeres, Dubai, abril de 2014.

Constitución de los Emiratos Árabes Unidos, Secretaría General del Consejo Nacional Federal, Dubai, 2015

EL-DIN AHMED, W. H., "Empoderamiento político de las mujeres árabes", Centro de investigación prometedora en investigación social y estudios de la mujer, Riad, 2016.

AL-DABBAGH. M. et al., "La experiencia parlamentaria de las mujeres emiratíes", Facultad de Dubai para Gestión Gubernamental, Dubai, 2009.

AMIRI, A. et al., "El papel de la mujer en el Consejo Nacional Federal", Consejo Nacional Federal, Dubai

Fundación de Mujeres de Dubai, Gobierno de los Emiratos Árabes Unidos, Informe estadístico de mujeres, Emiratos Árabes Unidos, Dubai, 2013.

MUTTAL, N. A., Empoderamiento de las Mujeres del Golfo, Oficina de Conocimientos Árabes, El Cairo, 2018. 
AL-GHARAIBEH, F. "Una exploración de la evolución de los roles de las mujeres en las sociedades del Consejo de Cooperación del Golfo" Fuente: Cuestiones de desarrollo social, Volumen 37, № 3 ,

Consorcio Internacional para Desarrollo Social, 2015. 